\title{
Erratum to: Evidence of selection signatures that shape the Persian cat breed
}

\author{
Francesca Bertolini $^{1}$ - Barbara Gandolfi ${ }^{2}$ Eui Soo Kim ${ }^{1}$ - Bianca Haase ${ }^{3}$. \\ Leslie A. Lyons ${ }^{2} \cdot$ Max F. Rothschild ${ }^{1}$
}

Published online: 23 March 2016

(C) Springer Science+Business Media New York 2016

\section{Erratum to: Mamm Genome \\ DOI 10.1007/s00335-016-9623-1}

Unfortunately, Table 3 in the original publication of the article was not published with the color notations. As stated by the caption, the table should be colored to underline the most frequent haplotypes in the Persian breed and to detect the same haplotype in the Persian derived breeds. The colored version of the table is published with this erratum.

The online version of the original article can be found under doi:10.1007/s00335-016-9623-1.

Max F. Rothschild

mfrothsc@iastate.edu

Francesca Bertolini

fbert@iastate.edu

1 Department of Animal Science, Iowa State University, Ames, IA, USA

2 Department of Veterinary Medicine and Surgery, University of Missouri, Columbia, USA

3 Faculty of Veterinary Science, University of Sydney, Sydney, Australia 
Table 3 Haplotype frequency of Persian and derived breeds

\begin{tabular}{|c|c|c|c|c|c|c|c|c|}
\hline & Persian & & British Shorthair & & Selkirk Rex & & Scottish Fold & \\
\hline & Haplotype & $\%$ & Haplotype & $\%$ & Haplotype & $\%$ & Haplotype & $\%$ \\
\hline \multirow[t]{3}{*}{$\begin{array}{l}\text { A2:4115484- } \\
42509338\end{array}$} & $\begin{array}{l}\text { AAAAGGGCGAGGGAGGACAGGC } \\
\text { CCAAGACGGCGGG }\end{array}$ & 82.5 & $\begin{array}{l}\text { AAAAGGGCGAGGGAGGACAGGC } \\
\text { CCAAGACGGCGGG }\end{array}$ & 34.8 & $\begin{array}{l}\text { AAAAGGGCGAGGGAGGACAG } \\
\text { GCCCAAGACGGCGGG }\end{array}$ & 36 & $\begin{array}{l}\text { AAAAGGGCGAGGGAGGACAGG } \\
\text { CCCAAGACGGCGGG }\end{array}$ & 41.7 \\
\hline & $\begin{array}{l}\text { AAAAGGGCGAGGGAGGACAGGC } \\
\text { CCAAGACGACGGG }\end{array}$ & 3.1 & $\begin{array}{l}\text { AAAAGGGCGAGGGAGGACAGGC } \\
\text { CAAAAACGACGGG }\end{array}$ & 21.7 & $\begin{array}{l}\text { AAAAGGGCGAGGGAGGACAG } \\
\text { GCCAAAAACGACGGG }\end{array}$ & 6 & $\begin{array}{l}\text { AAAAGGGCGAGGGAGGACAGG } \\
\text { CCAAAAACGACGGG }\end{array}$ & 10.8 \\
\hline & $\begin{array}{l}\text { AAAAGGGCGAGGGAGGACAGGC } \\
\text { CAAAAACGACGGG }\end{array}$ & 3.1 & $\begin{array}{l}\text { AAAGAAGCGCGGAGAGACAGGC } \\
\text { CCAGGAAGGCGGG }\end{array}$ & 8.7 & $\begin{array}{l}\text { AAGAGGGCGAGGGAGGACA } \\
\text { GGCCCAAGACGGCAGG }\end{array}$ & 8 & $\begin{array}{l}\text { AAAAGGGCGAGGGAGGACAGG } \\
\text { CCAAAAACGGCGGG }\end{array}$ & 9.2 \\
\hline \multirow[t]{3}{*}{$\begin{array}{l}\text { A2:44595242- } \\
45638786\end{array}$} & $\begin{array}{l}\text { GACAAAGAACAGCGAAAAGACG } \\
\text { CGAGA }\end{array}$ & 72.5 & $\begin{array}{l}\text { GACAAAGAACAGCGAAAAGACG } \\
\text { CGAGA }\end{array}$ & 41.3 & $\begin{array}{l}\text { GACAAAGAACAGCGAAAAGA } \\
\text { CGCGAGA }\end{array}$ & 20 & $\begin{array}{l}\text { GACAAAGAACAGCGAAAAGAC } \\
\text { GCGAGA }\end{array}$ & 16.2 \\
\hline & $\begin{array}{l}\text { GACAAAGAACAGCGAAAAGACAA } \\
\text { GAAA }\end{array}$ & 4.4 & $\begin{array}{l}\text { GCCAGAGAGCAGCGAAAAGACG } \\
\text { CGGGA }\end{array}$ & 6.5 & $\begin{array}{l}\text { GCCAGAGAGAAGCGAAAAGA } \\
\text { AGCGGGA }\end{array}$ & 10 & $\begin{array}{l}\text { GCCAGAGAGAAGCGAAAAGAA } \\
\text { GCGGGA }\end{array}$ & 9.9 \\
\hline & $\begin{array}{l}\text { GACAAAGAACAGCGAAAAGAAGC } \\
\text { GGGA }\end{array}$ & 4.4 & $\begin{array}{l}\text { GACAGAGAACAGCGAAAAGACG } \\
\text { CGAGA }\end{array}$ & 6.5 & $\begin{array}{l}\text { GCCAGAGAGAAGCGAAAAGA } \\
\text { CGCGAGA }\end{array}$ & 8 & $\begin{array}{l}\text { GACAAAGAACAGCGAAAAGAC } \\
\text { AAGAAA }\end{array}$ & 8.3 \\
\hline \multirow[t]{3}{*}{$\begin{array}{l}\text { C2:127112224- } \\
128268366\end{array}$} & $\begin{array}{l}\text { GGGACAAGGGGGCAGAGACGAA } \\
\text { AGACAGAA }\end{array}$ & 73.1 & $\begin{array}{l}\text { GGGACAAGGGGGCAGAGACGAA } \\
\text { AGACAGAA }\end{array}$ & 52.2 & $\begin{array}{l}\text { GGGACAAGGGGGCAGAGAC } \\
\text { GAAAGACAGAA }\end{array}$ & 52 & $\begin{array}{l}\text { GGGACAAGGGGGCGGGGACG } \\
\text { AAAGACAGAA }\end{array}$ & 19.4 \\
\hline & $\begin{array}{l}\text { GGGACAAGGGGGCGGGGACGA } \\
\text { AAGACAGAA }\end{array}$ & 10.3 & $\begin{array}{l}\text { GGGACAAGGGGGCGGGGACGA } \\
\text { AAGACAGAA }\end{array}$ & 17.4 & $\begin{array}{l}\text { GGGGCAAGGGGGCAGAGCA } \\
\text { GAAAGACAGAA }\end{array}$ & 12 & $\begin{array}{l}\text { GGGACAAGGGGGCAGAGACGA } \\
\text { AAGACAGAA }\end{array}$ & 13.7 \\
\hline & $\begin{array}{l}\text { GGAACAAAGAGACAGAGCAGAC } \\
\text { AGAAAGAA }\end{array}$ & 3.8 & $\begin{array}{l}\text { GGAACAAAGAGACAGAGCAGAC } \\
\text { AGAAAGAA }\end{array}$ & 8.7 & $\begin{array}{l}\text { GGGACAAGGGGGCGGGGAC } \\
\text { GAAAGACAGAA }\end{array}$ & 4 & $\begin{array}{l}\text { GGGGCAAGAGGGAAGAGACGA } \\
\text { ACAGCGGAA }\end{array}$ & 9.2 \\
\hline
\end{tabular}

The three most frequent haplotypes for each region in each breed are reported. For each region, the most frequent haplotypes in Persians is colored with red (region A2: 41154844-42509338), yellow (region A2: 44595242-45638786) and green (region C2: 127112224-128268366). The respective colors were used to detect the same haplotype in the Persian derived breeds 